\title{
Modeling capabilities of unsteady RANS for the simulation of turbulent swirling flow in an annular bluff-body combustor geometry
}

\author{
Yang Zhang ${ }^{1}$, Maarten Vanierschot ${ }^{1}$ \\ ${ }^{a}$ KU Leuven, Mechanical Engineering Technology TC, Group T Leuven Campus, Andreas Vesaliusstraat 13, B-3000 Leuven, \\ Belgium
}

\begin{abstract}
In this paper, we investigate the capabilities of the unsteady Reynolds-averaged Navier-Stokes (URANS) equations for capturing the helical structures found in a three-dimensional, incompressible and isothermal annular swirling jet undergoing vortex breakdown. The flow topology is representative of a bluff-body combustor operating at a Reynolds number $R e=8500$ and swirl number $S=0.39$. As a turbulence model, the SSG Reynolds stress model (RSM) was chosen. The numerical simulation is validated by means of tomographic particle image velocimetry measurements of the same flow configuration. To detect the coherent structures in the flow field, the recently introduced spectral proper orthogonal decomposition technique is adopted, which gives both temporal and spatial information on the large scale coherent structures. The spectral analysis of the sampled velocity signals identified a precessing vortex core with a frequency of $24.3 \mathrm{~Hz}$. In particular, two different large-scale helical flow structures are identified: a single and a double helix. Both of them are wound in the counter-swirl direction and wrapped around the central breakdown bubble. Those findings are quite similar to recent experimental tomographic particle image velocimetry results (Vanierschot et al., Phys. Rev. Fluids (2018) \& J. Fluid Mech. (2020)). This study demonstrates that the unsteady RANS approach with RSM is able to predict the coherent structures found in an annular swirling jet undergoing vortex breakdown both temporally and spatially with reasonable accuracy and this approach can hence be used in the design of bluff-body combustors, where a multi-dimensional parameter space may require many simulations to find the optimal design.

Keywords: Vortex breakdown; Precessing Vortex Core; Unsteady Reynolds-averaged Navier-Stokes simulation; Spectral proper orthogonal decomposition
\end{abstract}

${ }^{*}$ Corresponding author 


\section{Introduction}

Turbulent swirling flows are ubiquitous not only in nature, such as in tornadoes and typhoons, but also in technical engineering applications, for instance, the rotational motion in cyclone separators, heat exchangers, and gas turbines. Particularly, in combustion systems, swirl is added to the flow to enhance the fuel-air mixing rate and to reduce the emission of nitrogen oxides by means of a recirculation zone, which is caused by the centrifugal effects or adverse pressure gradients occurring above a certain level of the swirl intensity [? ]. Another prevalent feature in this type of flow is the so-called "vortex breakdown" phenomenon, which refers to an abrupt change in the structure of the vortex core. Much attention has been paid to the vortex breakdown phenomenon since it was reported by Peckham and Atkinson [? ]. Over the past several decades, at least seven different forms of vortex breakdown have been found, and the two most common ones among them are the nearly axisymmetric bubble type and the asymmetrical spiral type. Earlier studies mainly focused on the origins of vortex breakdown and several different theories have been introduced, such as those relating to wave phenomena and hydrodynamic instability, but there is not yet a unifying one accepted by the community. For a comprehensive review of vortex breakdown, we refer interested readers to the papers of Escudier [?] and Lucca-Negro and O'Doherty [? ] for more details.

The annular jet flow, i.e., fluid flow through a coaxial cylindrical passage, is widely used in bluff-body combustors. According to the experimental investigations by Ko and Chan [? ], the flow field of annular jets can be divided into three different regimes: the initial merging, the intermediate and the fully merged zone. In addition to the outer shear layer in an ordinary round jet, the channel of the annular jet also introduces an inner shear layer between the jet and the central recirculation zone. This recirculation zone introduced by flow separation behind the bluff-body is usually adopted to stabilize the flame in combustors [? ]. Both inner and outer shear layers would bring about shear-induced instabilities of the Kelvin-Helmholtz type. Owing to the imposed swirl, the flow dynamics in the system are rather complicated. When a critical swirl number is reached, the vortex breakdown bubble appears and this recirculation zone displaces the central vortex core resulting in a precessing vortex core (PVC) [? ]. Moreover, the vortex breakdown bubble interacts with the PVC for its precession around the jet axis.

As the oscillations of the PVC or other large-scale helical flow structures can amplify and lead to a feedback mechanism with acoustic modes in premixed combustion systems [? ? ], many studies have been conducted to explain the occurrence and to detect the manner of these coherent structures. The numerical simulation at low Reynolds number showed that spiral vortex breakdown might be interpreted as a nonlinear global mode which develops on the axisymmetric breakdown state [? ]. Experimental investigations by Liang 
et al. [? ] revealed that single and double helical structures could coexist with the single one being dominant after vortex breakdown. The stability analyses demonstrated that both single and double vortex breakdown are bifurcations from the axisymmetric bubble breakdown solution [? ]. Recently, Vanierschot et al. [? ] measured the velocity fields in a turbulent annular swirling jet using tomographic particle image velocimetry, and a double helix vortex breakdown structure which is rarely reported in the turbulent regime was detected by the phase averaging method. In addition, both single and double helical structures were identified by the spectral proper orthogonal decomposition method, and a global stability analysis of the mean flow field revealed that they are the two dominant global modes [? ].

Although Reynolds-averaged Navier-Stokes (RANS) is not as accurate as scale resolved simulations (SRS), large eddy simulations (LES) or direct numerical simulations (DNS), it is widely adopted to model turbulent flows as it has low computational cost and can be used for highly complex flow geometries [? ]. There have been many studies related to RANS models in simulating turbulent swirling flows, both in steady and unsteady cases [? ? ? ? ? ? ? ? ]. Despite the fact that the $k-\varepsilon$ model overpredicts the decay of swirl because it assumes an isotropic turbulent viscosity, Guo et al. [? ] simulated the turbulent swirling flows in axisymmetric sudden expansions using a standard $k-\varepsilon$ turbulence model, and the results showed that the calculated mean flow field and the predicted precession frequency of the PVC were in close agreement with the reported values in the literature. Wegner et al. [? ] evaluated the capability of unsteady RANS to predict the PVC phenomenon in a movable block swirl device based on LES data and experiments. Firstly, they used a standard $k-\varepsilon$ model and found that the flow instability decayed as the simulation progressed and obtained a steady-state solution. Then, the Reynolds stress model was chosen and predicted the PVC phenomenon both qualitatively and in parts quantitatively. Jochmann et al. [? ] presented the results of numerical predictions of a spiral type vortex breakdown with a fixed precession frequency in a realistic gas turbine combustor using $k-\varepsilon$ and SSG Reynolds stress models. Compared with particle image velocimetry and laser Doppler anemometry measurements from experiments, they concluded that both turbulence models were capable of yielding the spatial and temporal dynamics of the flow. Dunham et al. [? ] compared the performance of unsteady RANS with a $k-\varepsilon$ model and LES for air swirl fuel injectors, and both methodologies captured the gross time-mean features of strongly swirling confined flows in reasonable agreement with experiments. However, the spectral analysis showed that unsteady RANS predicted the presence of coherent structures with incorrect amplitudes and frequencies. The work of RamÍrez and Cortés [? ] showed the comparison of a standard $k-\varepsilon$ model and Reynolds stress model for the simulations of turbulent flow in a swirl combustor. Their results were quite convincing and compared reasonably with the experimental literature in terms of the 
predicted PVC and pulsating inner recirculation zone. Miltner et al. [? ] presented a detailed analysis of the flow of a straight and slightly rotating turbulent free jet with various turbulence models both numerically and experimentally, and they found that inaccuracies could be introduced if an inappropriate turbulence model is applied although numerical simulation is a suitable way for the accurate description of the analyzed flow type. Rukes et al. [? ] assessed the applicability of unsteady RANS to supplement experimental approaches in the investigations of the dominant coherent structures in a swirling jet. The results from the second order Reynolds stress model were qualitatively and quantitatively in line with experimental findings. Using a realizable $k-\varepsilon$ model, Saqr et al. [? ] investigated the effect of swirl level on heat transfer and entropy generation in a non-isothermal turbulent pipe flow and good agreement with experimental data was found.

All these studies show that the URANS method in combination with the $k-\varepsilon$ family or Reynolds stress model are capable of capturing the highly unsteady flow pattern in turbulent swirling flows. However, there are some drawbacks, like the inability to predict multiple frequency phenomena [? ]. Studies by Brown et al. [? ? ] on industrial swirling flows showed that the Scale Resolved Model performed much better in predicting those dynamical phenomena compared to URANS. Also, the LES approach has been demonstrated to be suitable for predicting the turbulent flow field and mixing characteristics of a complex swirled flow of a generic laboratory-scaled combustor similar to those used for oxy-fuel combustion [? ]. However, the introduction of wall modelling, to reduce the number of grid cells, interacts with the laminarto-turbulent transition. Hence, a fully resolved boundary layer is needed to avoid those effects, which would be computationally much more expensive.

Although the literature on round swirling jets is plentiful, only few numerical simulations of turbulent annular vortex flows have been conducted. Garca-Villalba et al. [? ] analyzed coherent structures in the near field of a turbulent unconfined annular swirling jet using the LES method. The vortex and turbulence decay phenomena that take place in an annular pipe flows were simulated using different RANS models and the main characteristics of the flow such as vortex structure and recirculation zone were investigated by Saqr et al. [? ]. The LES of annular swirling flow in confined jet configurations demonstrated that small flow modifications could trigger bifurcation and hysteresis close to critical flow conditions[? ]. Mullyadzhanov et al. studied the transient dynamics of flow in a rotating-pipe swirl burner by LES, which suggested that the sudden hysteretic change of the regime observed depends on whether the thermal effects will overrule the inertia of the strong near-nozzle vortex structures [? ]. Gritskevich et al. investigated the flow near a round exhaust channel screened by an annular swirled jet using the SST model to design effective systems of exhaust ventilation of lowered energy capacity [? ]. The LES conducted by Ryzhenkov and Mullyadzhanov [? 
] showed the flow dynamics in the near field of a swirling turbulent annular jet. Also, the LES study of the annular non-swirling flow revealed that the flow pattern would be similar to the low-swirling jets for particular Reynolds number and inner shear layer thickness, and the flow instability could lead to the formation of spiral structures [? ]. Mansouri and Boushaki [? ] used the delayed detached eddy simulation to model the turbulent flow field of a non-premixed swirl burner and to compare the results with those from stereo particle image velocimetry measurements. Despite the fact that SRS, RANS/LES or LES can predict the dynamical phenomena in swirling flows much better compared to URANS, the computational effort is significantly higher and therefore in the design process of combustors, where a lot of parameters might need to be changed for the optimal design and working conditions, the computational time might become excessively high.

Recently, tomographic particle image velocimetry experiments of an annular swirling jet at a Reynolds number of 8500 at moderate swirl showed the presence of a double helical structure in the flow field [? ? ], which is rarely observed in turbulent flows. This double helical structure could also be captured by unsteady RANS modeling of the same flow configuration using an RNG turbulence model [? ]. However, comparison with experimental data showed moderate agreement of the obtained flow fields : the downstream position of the vortex breakdown bubble and the breakup location of both helices was much closer to the nozzle and the decay of the swirl velocity was overpredicted. Therefore, in this work, we test the capabilities of URANS with a more advanced turbulence model, the Reynolds stress model (RSM). Although plenty studies show that the RSM model can handle the anisotropic flow field of swirling flows very well, very little information is given whether it could also capture the presence of multiple dominant periodic structures, their mutual interaction and their influence on the second order statistics of the flow field. Therefore, this study aims to assess the capability of RSM to predict the multiple coherent structures found in a turbulent annular swirling jets undergoing vortex breakdown and their interaction. Firstly, the simulations are validated by detailed experimental data. Secondly, we apply a modal decomposition approach to identify the coherent structures and their dynamics and compare results with those from literature. The rest of the paper is arranged as following: the turbulence model, computational grids, and numerical scheme are introduced in Sec. ??. Sec. ?? contains the main results of the unsteady RANS simulation: Sec. ??, general features of the mean flow field; Sec. ??, temporal dynamics of the PVC; Sec. ??, the spectral proper orthogonal decomposition analysis of the velocity fields; and Sec. ??, the detected helical structures. Finally, we summarize our findings and present the conclusions in Sec. ??. 


\section{Computational setup}

\subsection{Choice of turbulence model}

Based on the aforementioned unsteady RANS simulations, we attempted to use a standard $k-\varepsilon$ model to simulate the turbulent annular swirling jet flow in the current work. However, a steady-state was acquired as the simulation progressed, which is similar to the works reported by Wegner et al. [? ] and Rukes et al. [? ]. As such, the precessing vortex core could not be captured, most probably due to the dissipative character of the turbulence models. Further studies showed that other turbulence models, such as $k-\omega$ SST, gave a similar result as well. Finally, the second-order SSG Reynolds stress model [? ] was chosen following the simulations executed in Refs. [? ? ? ? ], in which the transport equation for the Reynolds stress $\overline{u_{i}^{\prime} u_{j}^{\prime}}$ needs to be modelled:

$$
\frac{\partial \overline{u_{i}^{\prime} u_{j}^{\prime}}}{\partial t}+\bar{u}_{k} \frac{\partial \overline{u_{i}^{\prime} u_{j}^{\prime}}}{\partial x_{k}}=-\frac{\partial T_{k i j}}{\partial x_{k}}+\mathcal{P}_{i j}+\mathcal{R}_{i j}-\varepsilon_{i j}+\nu \frac{\partial^{2} \overline{u_{i}^{\prime} u_{j}^{\prime}}}{\partial x_{k}^{2}}
$$

Here, the Reynolds-stress flux $T_{k i j}$, the production tensor $\mathcal{P}_{i j}$, the pressurerate-of-strain tensor $\mathcal{R}_{i j}$, and the dissipation tensor $\varepsilon_{i j}$ are given respectively as follows:

$$
\begin{gathered}
T_{k i j}=\overline{u_{i}^{\prime} u_{j}^{\prime} u_{k}^{\prime}}+\frac{\overline{p^{\prime} u_{i}^{\prime}}}{\rho} \delta_{j k}+\frac{\overline{p^{\prime} u_{j}^{\prime}}}{\rho} \delta_{i k}, \\
\mathcal{P}_{i j}=-\overline{u_{i}^{\prime} u_{k}^{\prime}} \frac{\partial \bar{u}_{j}}{\partial x_{k}}-\overline{u_{j}^{\prime} u_{k}^{\prime}} \frac{\partial \bar{u}_{i}}{\partial x_{k}}, \\
\mathcal{R}_{i j}=\frac{\overline{p^{\prime}}}{\rho}\left(\frac{\partial u_{i}^{\prime}}{\partial x_{j}}+\frac{\partial u_{j}^{\prime}}{\partial x_{i}}\right), \\
\varepsilon_{i j}=2 \nu \frac{\partial u_{i}^{\prime}}{\partial x_{k}} \frac{\partial u_{j}^{\prime}}{\partial x_{k}} .
\end{gathered}
$$

The production term $\mathcal{P}_{i j}$ and the last viscous diffusion term $\nu \frac{\partial^{2} \overline{u_{i}^{\prime} u_{j}^{\prime}}}{\partial x_{k}^{2}}$ do not contain any unclosed expressions and thus they are resolved. The turbulent transport term $T_{k i j}$ could be modeled by a gradient-diffusion 
Table 1: Model constants in SSG Reynolds stress model

\begin{tabular}{lllllllllll}
\hline \hline$C_{1}$ & $C_{1}^{*}$ & $C_{2}$ & $C_{3}$ & $C_{3}^{*}$ & $C_{4}$ & $C_{5}$ & $C_{\varepsilon}$ & $C_{\varepsilon 1}$ & $C_{\varepsilon 2}$ & $C_{s}$ \\
\hline 3.4 & 1.8 & 4.2 & 0.8 & 1.3 & 1.25 & 0.4 & 0.15 & 1.44 & 1.92 & 0.25 \\
\hline
\end{tabular}

model [?]:

$$
T_{k i j}=-C_{s} \frac{k}{\varepsilon} \overline{u_{k}^{\prime} u_{l}^{\prime}} \frac{\partial \overline{u_{i}^{\prime} u_{j}^{\prime}}}{\partial x_{l}}
$$

The pressurerate-of-strain tensor $\mathcal{R}_{i j}$ in the SSG Reynolds stress model is given by:

$$
\begin{aligned}
\mathcal{R}_{i j}=-\left(C_{1} \varepsilon+\right. & \left.C_{1}^{*} \mathcal{P}\right) b_{i j}+C_{2} \varepsilon\left(b_{i k} b_{k j}-\frac{1}{3} b_{k l} b_{k l} \delta_{i j}\right)+\left(C_{3}-C_{3}^{*}\left(b_{k l} b_{k l}\right)^{1 / 2}\right) k \bar{S}_{i j} \\
& +C_{4} k\left(b_{i k} \bar{S}_{j k}+b_{j k} \bar{S}_{i k}-\frac{2}{3} b_{k l} \bar{S}_{k l} \delta_{i j}\right)+C_{5} k\left(b_{i k} \bar{W}_{j k}+b_{j k} \bar{W}_{i k}\right)
\end{aligned}
$$

where $\mathcal{P}=\frac{1}{2} \mathcal{P}_{i i}=-\overline{u_{k}^{\prime} u_{i}^{\prime}} \frac{\partial \bar{u}_{i}}{\partial x_{k}}, b_{i j}=\frac{\overline{u_{u}^{\prime} u_{j}^{\prime}}-\frac{2}{3} k \delta_{i j}}{2 k}$ is the anisotropy of Reynolds stress, $\bar{S}_{i j}=\frac{1}{2}\left(\frac{\partial \bar{u}_{i}}{\partial x_{j}}+\frac{\partial \bar{u}_{j}}{\partial x_{i}}\right)$ and $\bar{W}_{i j}=\frac{1}{2}\left(\frac{\partial \bar{u}_{i}}{\partial x_{j}}-\frac{\partial \bar{u}_{j}}{\partial x_{i}}\right)$ are the mean rate of strain tensor and mean vorticity tensor, respectively. The dissipation tensor $\varepsilon_{i j}$ is assumed to be local isotropic:

$$
\varepsilon_{i j}=\frac{2}{3} \varepsilon \delta_{i j}
$$

and the standard model equation for $\varepsilon$ introduced by Hanjalić and Launder [? ] is used,

$$
\frac{\partial \varepsilon}{\partial t}+\bar{u}_{i} \frac{\partial \varepsilon}{\partial x_{i}}=\frac{\partial}{\partial x_{i}}\left(C_{\varepsilon} \frac{k}{\varepsilon} \overline{u_{i}^{\prime} u_{j}^{\prime}} \frac{\partial \varepsilon}{\partial x_{j}}\right)+C_{\varepsilon 1} \frac{\mathcal{P} \varepsilon}{k}-C_{\varepsilon 2} \frac{\varepsilon^{2}}{k} .
$$

The values of the model constants used in the SSG model depicted above are listed in Table ??.

\subsection{Computational domain, boundary conditions and grid}

Figure ?? shows a cross section of the computational flow domain used in this study, which is axisymmetric about the $y$-axis of the coordinate system. The numerical domain is designed to emulate the experimental set-up described in [? ], which means that the annular channel has the same dimensions in both cases. The working fluid, which is water, flows through an annular tube firstly and then mixes with the ambient medium in a large cylindrical domain whose length and diameter are $15 D_{o}$ and $16 D_{o}$, respectively. Here, $D_{o}=27 \mathrm{~mm}$ is the outer diameter of the annular pipe, whereas the length $L$ and inner diameter $D_{i}$ 
of it are $54 \mathrm{~mm}$ and $18 \mathrm{~mm}$, respectively. There are several different ways to generate a swirling flow by imparting azimuthal momentum to an axial through-flow, such as adjustable guided vanes [? ], a rotating inlet tube [? ], a rotating perforated plate [? ], and so forth. In the experimental set-up, the swirl intensity was generated by 12 adjustable guide vanes [? ]. However, in the present work, the annular swirling jet flow is generated by setting an axial-tangential fluid entry for simplicity [? ], and the constant inlet axial and angular velocities are set to be $U_{0}$ and $\omega$ in the cross-streamplane, respectively. To avoid the formation of a large, unphysical recirculation zone in the outer region, the entrained fluid which is called a co-flow is fed in with a mild velocity $U_{c}=0.04 U_{0}$. This co-flow boundary condition was not presented in the experiment, and it is usually adopted in numerical simulations [? ].

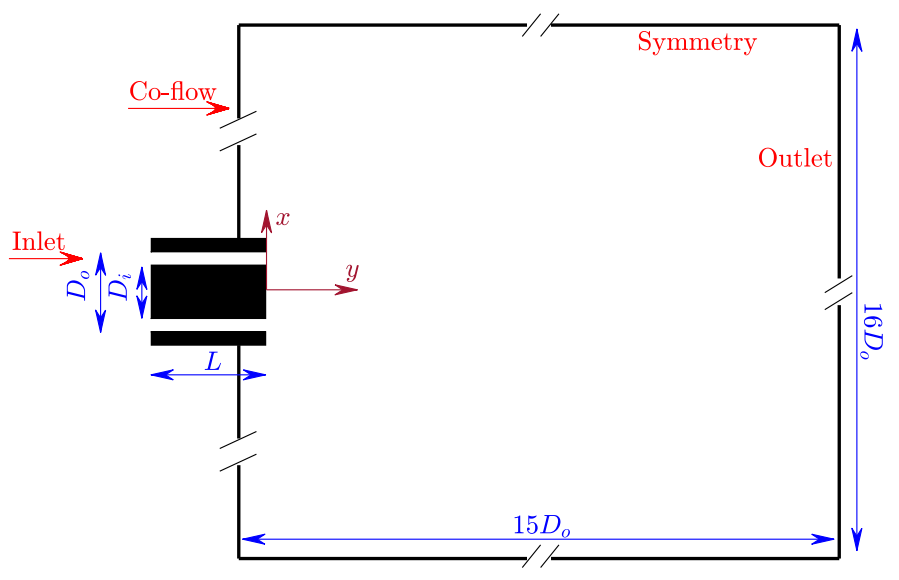

Figure 1: The schematic diagram of the computational flow domain used in the simulation.

The no-slip velocity boundary condition is applied to the inner and outer wall of the annular pipe, and the end wall of the central bluff-body. The zero gradient boundary condition for velocity is employed at the lateral wall of the unconfined domain and on the fluid leaving the computational domain. Ambient pressure is imposed for the far-field boundary, whereas zero gradient boundary conditions are applied to all other boundaries including the inlet, co-flow, solid surfaces, and the lateral wall of the computational domain. For the Reynolds stresses, Dirichlet boundary conditions are given at the inlet and the walls of the annular coaxial tube, and a Neumann boundary condition is adopted at the lateral wall and outlet of the domain. The dissipation rate is set with a fixed value boundary condition (calculated by $\varepsilon=C_{\mu}^{3 / 4} \frac{k^{3 / 2}}{l}$ from the turbulence model constant $C_{\mu}=0.09$, and the turbulent length scale $l=0.07 D_{h}$ based on the hydraulic diameter $D_{h}$ of the annular pipe), and a zero gradient condition across all other faces of the computational domain. 

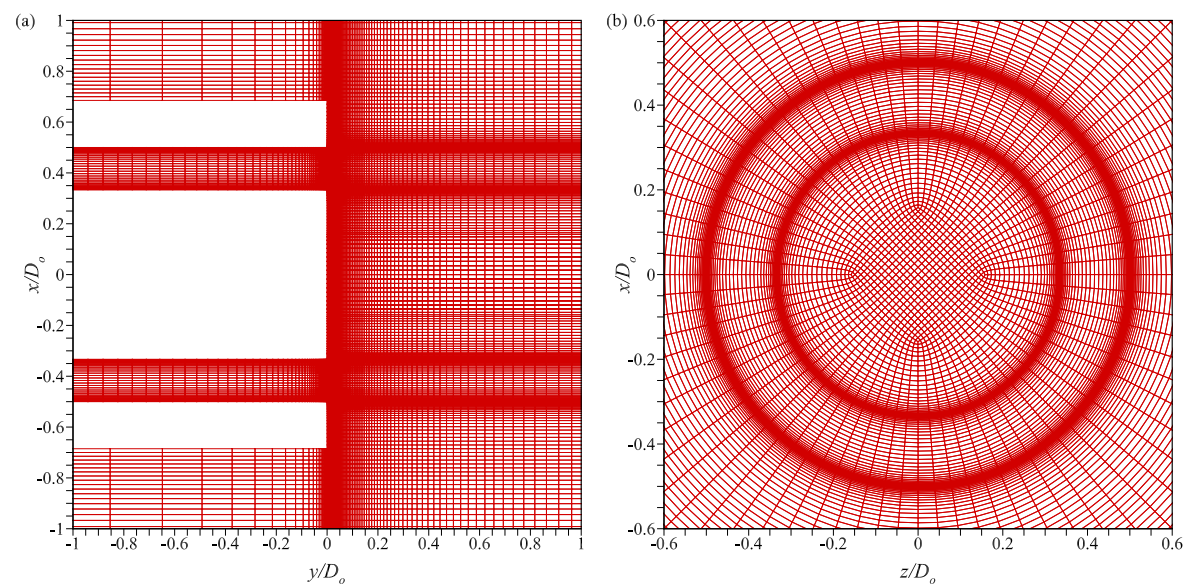

Figure 2: Computational grid close to the outlet of the annular pipe: (a) cross section in the $x-y$ plane, (b) axisymmetric plane perpendicular to the $y$-axis of the geometry.

The computational domain shown in figure ?? is meshed in Pointwise V18.2R2 using a structured multiblock approach. Two mesh resolutions, the coarse one with 1.77 million cells and the other finer one with 4.16 million cells, are generated to evaluate the grid convergence. Figures ??(a,b) display the computational grid close to the nozzle exit. All grid cells are hexahedral with high quality regarding aspect ratio, nonorthogonality, and skewness. The grid size is smaller adjacent to the walls to resolve the viscous sublayer as no wall functions are used in the current study. The results discussed in this work are obtained using the open-source computational fluid dynamics package OpenFOAM v1706, in which the finite volume method is implemented to discretize the incompressible Navier-Stokes equations. The temporal discretization is second-order implicit Euler. The spatial discretizations are second-order upwind for the convective fluxes and central differences for the diffusive fluxes, respectively. The pressure-velocity coupling is obtained using the PIMPLE algorithm, which is a combination of PISO (pressure implicit with splitting of operators) and SIMPLE (semi-implicit method for pressure-linked equations) algorithms. The geometric-algebraic multi-grid (GAMG) approach with simplified diagonal-based incomplete Cholesky (DIC) smoother is used to treat the pressure equation. The Gauss-Seidel iterative method is used for the velocity and turbulent quantities. Numerical accuracy is set to double precision and the initial conditions are chosen to be uniform. The time-dependent simulation is started from a steady calculation executed at the initial stage. A fixed time step of $6 \times 10^{-5}$ is chosen to guarantee the solver stability during the simulation and to ensure a sufficiently high temporal resolution. Then the unsteady simulation is started for several dimensionless units $t^{*}$ (normalization of the time $t$ as $U_{0} t / D_{o}$ ) to eliminate the effect of the initial transient regime. The sampling of the variables is performed after reaching the periodic state during an interval of $400 t^{*}$, which 
is long enough to obtain converged statistical values, at least in the near field of the jet exit. To ensure sufficient near-wall mesh resolution, the $y^{+}$value is $1.5(0.8)$ on average and does not exceed $3.6(2.4)$ with a maximum Courant-Friedrichs-Lewy (CFL) number of 3.9 (3.0) and a mean CFL number of 0.0014 (0.0011) for the coarse (fine) grid case.

Figure ??(a) presents the normalized mean streamwise velocity along the jet axis obtained from the two different mesh resolutions. The velocity near the nozzle is positive and becomes negative further downstream due to vortex breakdown, resulting in the typical velocity profile along the central axis for an annular swirling jet in that swirl number range [? ]. It is shown that there is no qualitative difference between the results from the two numerical grids. The maximum quantitative difference between the two different grids is less than $3 \%$ of the inlet axial velocity $U_{0}$. Also, the location of the stagnation point $y / D_{o} \approx 0.82$ indicated by the arrow predicted by the numerical simulation is quite close to the experimental value of 0.9 [? ]. The comparison of normalized mean axial $\bar{U}_{y} / U_{0}$ and tangential $\bar{U}_{z} / U_{0}$ velocity for two different numerical grids at four different axial locations are given in figures ??(b) and ??(c), respectively. It can be seen that the velocity profiles along the radial direction are almost the same for four different axial positions in the near field of the annular jet as well. Hence, it could be concluded that the results for these solvers and numerical schemes are virtually independent of the mesh resolution. The results discussed in the rest of the paper are acquired from the coarse grid.

\subsection{Control parameters}

The swirling jet flow can be characterized by two control parameters. The first one is the Reynolds number

$$
R e=U_{0} D_{h} / \nu
$$

which measures the turbulence intensity based on the axial inlet velocity $U_{0}=0.94 \mathrm{~m} / \mathrm{s}$, and the hydraulic diameter $D_{h}=9 \mathrm{~mm}$ of the annular tube. The working fluid is water with kinematic viscosity $\nu=$ $1.004 \times 10^{-6} \mathrm{~m}^{2} / \mathrm{s}$. The other parameter is the swirl number

$$
S=\frac{M_{\theta}}{M_{y}\left(D_{o} / 2\right)},
$$


which is the ratio of the axial flux of tangential momentum

$$
M_{\theta}=\int_{\frac{D_{i}}{2}}^{\frac{D_{o}}{2}} 2 \pi \rho \bar{U}_{y} \bar{U}_{\theta} r^{2} d r
$$

to the axial flux of axial momentum

$$
M_{y}=\int_{\frac{D_{i}}{2}}^{\frac{D_{o}}{2}} 2 \pi \rho \bar{U}_{y}^{2} r d r
$$

divided by a characteristic radius $D_{o} / 2$, where $\bar{U}_{y}$ and $\bar{U}_{\theta}$ are the mean axial and azimuthal velocity measured at the end of the annular channel, respectively. The computed Reynolds number Re is around 8500 , and the swirl number $S$ is calculated as 0.39 . The isothermal simulation that satisfies the similarity criterion in the form of the Reynolds number carried out in this work makes it possible to investigate the fluid dynamics of combustion flow as the large scale structures found in both isothermal and combustion flows are very similar [? ? ].

\section{Results and discussion}

\subsection{Mean flow fields and experimental validation}

Figures ??(a,b) depict the mean axial and azimuthal velocity profiles at several different axial locations together with streamlines in the $x-y$ plane. It is found that the axial velocity profiles are wake-like downstream of the annular jet nozzle, which is similar to the observation in experimental studies [? ? ]. The first reason is due to flow separation behind the central bluff-body, which causes a central recirculation zone. In addition, because of the high swirl intensity near the jet nozzle, the central recirculation zone has a toroidal shape. The second recirculation zone comes from the recirculation bubble caused by vortex breakdown further downstream at $y / D_{o}>0.8$. This value is very close to the experimental value of $y / D_{o}=0.9[?]$ and much better than the value predicted by the RNG model $\left(y / D_{o}=0.4\right)[?]$. Figure ??(a) provides the normalized axial velocity profiles at different axial stations, which shows that the unsteady RANS velocity profiles in the immediate near field are in line with the measured profiles, except that the velocity around the jet center is overestimated (note that the experimental velocity profiles are a little asymmetric). Further downstream, the unsteady RANS velocity profiles in the jet center region are underestimated, which means that the size of the simulated vortex breakdown bubble is larger than the experimental one and the peak value of the tangential velocity on this plane occurring at a larger radius than seen in the experiment. Correspondingly, the normalized azimuthal velocity profiles at different axial stations are given in figure ??(b). It 

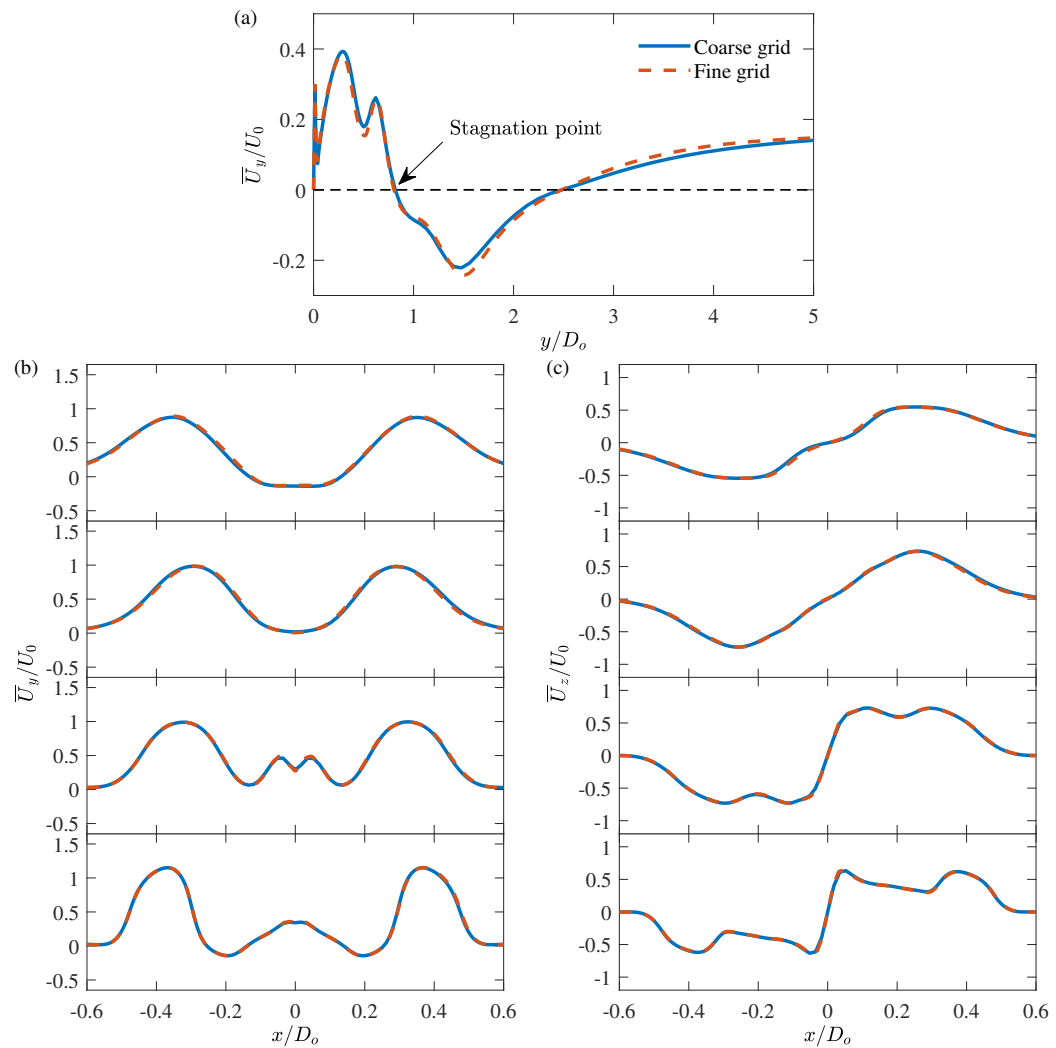

Figure 3: (a) The comparison of normalized mean axial velocity $\bar{U}_{y} / U_{0}$ along the axis of swirling jet obtained from the two different computational grids. The arrow indicates the location of the stagnation point $\left(y / D_{o} \approx 0.82\right)$, which agrees well with the experimental measurements by Percin et al. [? ]. (b) and (c) respectively present the comparison of normalized mean axial $\bar{U}_{y} / U_{0}$ and tangential $\bar{U}_{z} / U_{0}$ velocity for two different numerical grids at four different axial positions (from bottom to top $\left.y / D_{o}=0.2,0.4,0.8,1.2\right)$.
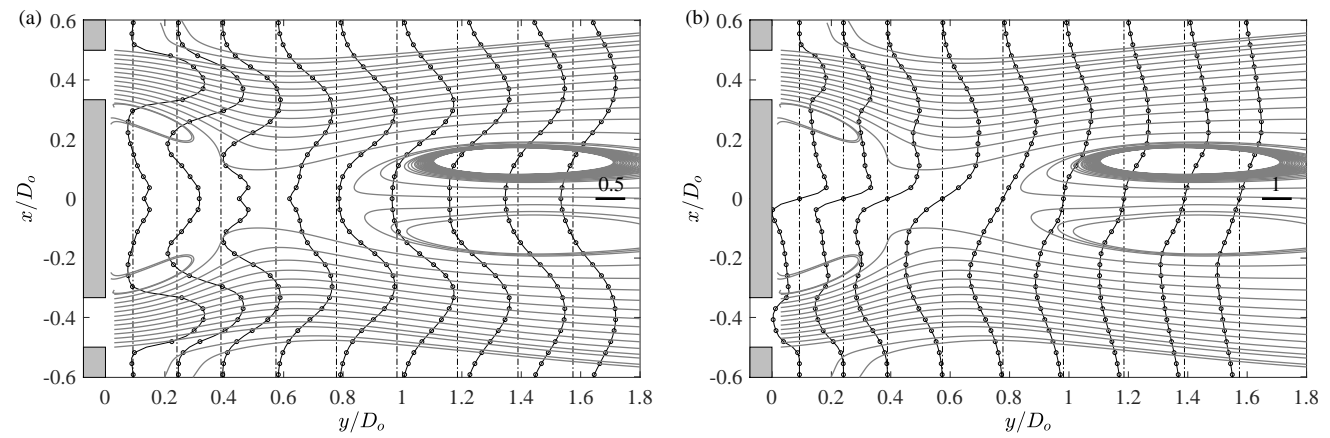

Figure 4: Time-averaged velocity profiles at different axial locations immediately downstream of the central bluff-body (a small section of the whole flow domain) normalized by $U_{0}$ : (a) mean axial velocity $\bar{U}_{y} / U_{0}$ and (b) mean tangential velocity $\bar{U}_{z} / U_{0}$. The grey lines represent streamlines of the mean flow in the plane. 
is shown that the numerically predicted and experimentally measured velocity profiles show good agreement in both magnitude and shape in the near field of the annular jet. Compared to the RNG model, the decay of azimuthal velocity is not overpredicted [? ]. On the other hand, the distribution of the Reynolds stresses immediately downstream of the bluff-body in the $x-y$ plane are given in figures ??(a-f) to compare with those provided by experimental studies (see for instance figure 8 in ref. [? ] for more detail). It is shown that the RSM model predicts the patterns of the normal stresses correctly when compared with the experimental measurements. The normal Reynolds stresses are highly anisotropic and large values exist in the inner shear layer of the bluff body wake, the outer shear layer of the jet and in the boundary of the vortex breakdown bubble. The RNG model can not capture this anisotropy as it assumes an isotropic turbulent viscosity. Also the shear forces in figures ??(d-f) show the same distribution as in the experiments.
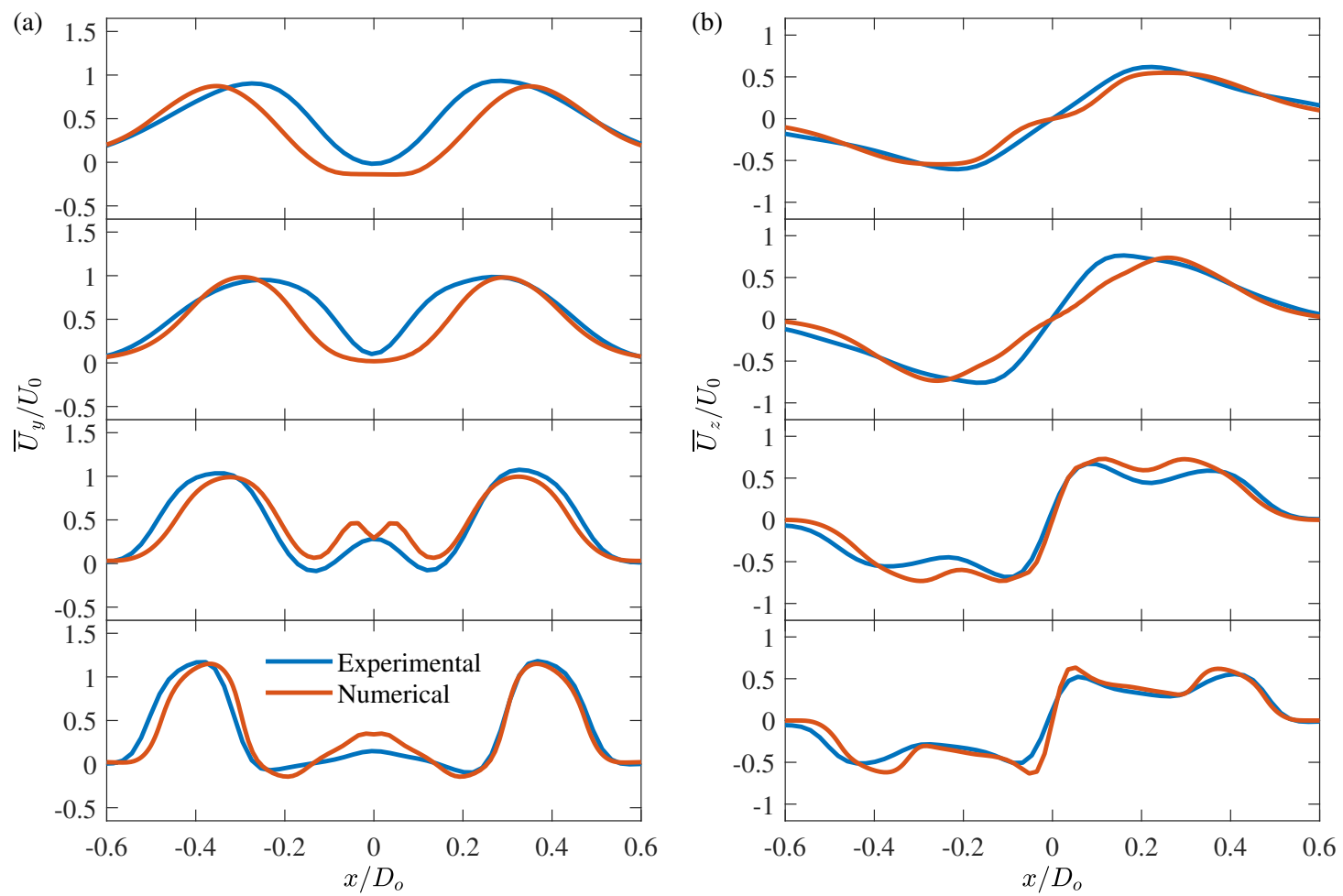

Figure 5: The comparison between experimentally and numerically obtained velocity profiles along radial direction at four different axial locations (from bottom to top $y / D_{o}=0.2,0.4,0.8,1.2$, respectively): (a) mean axial velocity $\bar{U}_{y} / U_{0}$, (b) mean azimuthal velocity $\bar{U}_{z} / U_{0}$.

\subsection{Temporal analysis}

To detect the frequency of the PVC, the values of the flow variables, including velocity components and pressure, are recorded every time step at several chosen locations in the flow field (for instance, the stagnation 

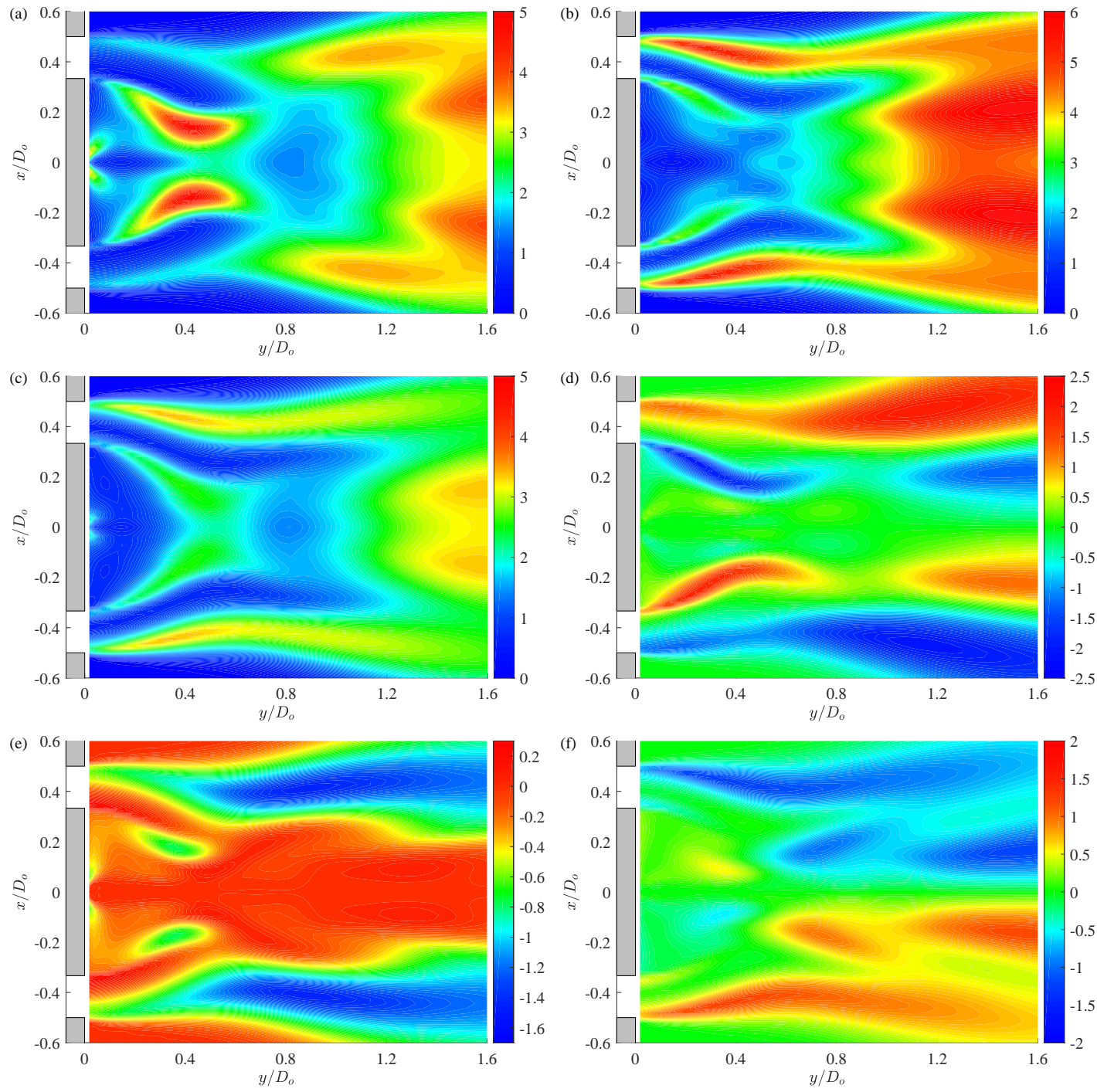

Figure 6: The normalized Reynolds stress components immediately downstream of the bluff-body in the $x-y$ plane: (a) $100 \times\left\langle\overline{u_{x}^{\prime} u_{x}^{\prime}}\right\rangle / U_{0}^{2}$, (b) $100 \times\left\langle\overline{u_{y}^{\prime} u_{y}^{\prime}}\right\rangle / U_{0}^{2}$, (c) $100 \times\left\langle\overline{u_{z}^{\prime} u_{z}^{\prime}}\right\rangle / U_{0}^{2}$, (d) $100 \times\left\langle\overline{u_{x}^{\prime} u_{y}^{\prime}}\right\rangle / U_{0}^{2}$, (e) $100 \times\left\langle\overline{u_{x}^{\prime} u_{z}^{\prime}}\right\rangle / U_{0}^{2}$, and (f) $100 \times\left\langle\overline{u_{y}^{\prime} u_{z}^{\prime}}\right\rangle / U_{0}^{2}$. 

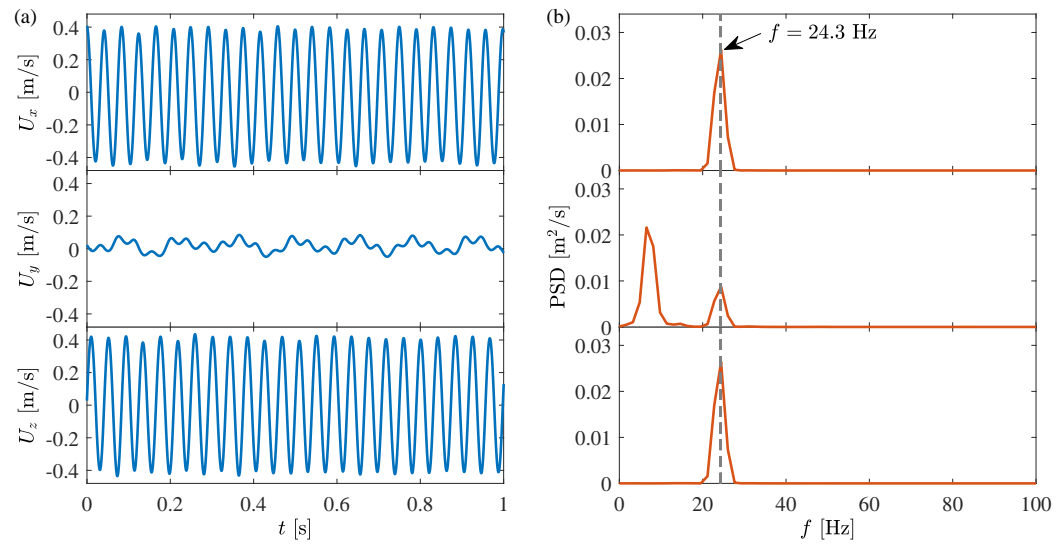

Figure 7: The sampled velocity signals at the stagnation point (a) and the corresponding power spectral density (PSD) distribution (b). Note that the PSD of axial velocity $U_{y}$ is multiplied by a factor of 100 for easy comparison. The arrow and the dashed line in figure (b) show that the PSD distributions of the three velocity components all have a peak with a frequency of $24.3 \mathrm{~Hz}$.

point and close to the inner and outer shear layers) during the computation of this time-dependent flow field. Typically, the monitored velocity variables at the stagnation point are plotted in figure ??(a). As unsteady RANS is only able to resolve the ensemble or phase-averaged coherent unsteadiness, the recorded signals are purely periodic. Additionally, at the stagnation point, the axial velocity magnitude is quite small when compared with the radial ones. Figure ??(b) illustrates the corresponding spectral information of the velocity components as shown in figure ??(a). Here, the power spectral density (PSD) of axial velocity is multiplied by a factor of 100 for clarity. It is shown that a dominant frequency about $24.3 \mathrm{~Hz}$ is predicted by the numerical simulation, at which all the spectral distributions have a peak. This value is very close to the value of $25 \mathrm{~Hz}$ obtained from the RNG model. Recently, the spiral type of vortex breakdown in an annular swirling jet with a precessing frequency of $28 \mathrm{~Hz}$ has been reported experimentally at the same Reynolds and swirl numbers by Vanierschot et al. [? ? ]. The result shows that there is a frequency shift of the PVC between the experimental value and the numerical one [? ]. This discrepancy is related to the inaccurate prediction of the azimuthal velocity profiles upstream of the vortex breakdown bubble as plotted in figure ??. In isothermal swirling flow, the frequency of the precessing vortex core is proportional to the tangential velocity gradient near the central axis [? ? ? ], which is underpredicted by the numerical simulations. The same dominant frequency of $24.3 \mathrm{~Hz}$ is obtained from the PSD of pressure and velocity components at other monitored points as well (not shown here). Apart from the frequency of PVC, the PSD of the streamwise velocity also shows a frequency peak of $7 \mathrm{~Hz}$, which refers to the oscillation of the vortex breakdown bubble in the axial direction. The RNG model is not able to capture this motion. 


\subsection{Spectral proper orthogonal decomposition analysis}

One of the key issues in this research field is to identify the coherent structures from turbulent flow fields, which involves the construction of empirical modes that dominate the flow dynamics. A suitable approach to extract the organized coherent large scale structure is the triple decomposition introduced by Hussain and Reynolds [? ], which has the following form:

$$
\boldsymbol{v}(\boldsymbol{x}, t)=\overline{\boldsymbol{v}}(\boldsymbol{x})+\widetilde{\boldsymbol{v}}(\boldsymbol{x}, t)+\boldsymbol{v}^{\prime}(\boldsymbol{x}, t) .
$$

In this formula, the instantaneous velocity signal $\boldsymbol{v}$ is decomposed into the mean value $\overline{\boldsymbol{v}}$, a phase-locked average part $\widetilde{\boldsymbol{v}}$, and a turbulent part $\boldsymbol{v}^{\prime}$. The coherent motions in turbulent flows can also be detected by means of energy-ranked proper orthogonal decomposition (POD) [? ]. In addition, there are several other decomposition methods other than the ones mentioned above, for instance, the frequency-ranked fast Fourier decomposition or dynamic mode decomposition [? ]. However, these methods are not applicable when coherent structures occur with low energies or multiple frequencies. The recently introduced spectral proper orthogonal decomposition (SPOD) approach allows us to detect the coherent structures both in spatial and in frequency domains especially when the flow dynamics are rather complicated [? ]. The formulation of the SPOD technique is given as follows:

$$
\boldsymbol{v}(\boldsymbol{x}, t)=\overline{\boldsymbol{v}}(\boldsymbol{x})+\sum_{i=1}^{N} a_{i}(t) \boldsymbol{\Phi}_{i}(\boldsymbol{x})
$$

in which the unsteady component is split into a collection of spatial modes $\boldsymbol{\Phi}_{i}$ and associated temporal coefficients $a_{i}$.

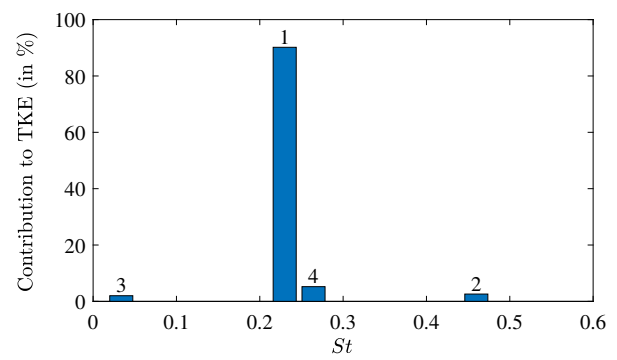

Figure 8: The contribution to turbulent kinetic energy (TKE) by the identified four SPOD mode pairs.

As shown before, the unsteady RANS simulation is only able to capture quasi-periodic flow features of turbulent fields. The postprocessing of sampled velocity fields shows that there are only four mode pairs 
extracted by SPOD and their energy content is depicted in figure ??. Each bar corresponds to one mode pair in the figure and these four ranked mode pairs are labeled with numbers to indicate the magnitude of their harmonic correlation. Here, the Strouhal number $S t$ is defined as $S t=f D_{h} / U_{0}$, where $f$ is the empirical mode's frequency. The SPOD results show that the first two harmonic mode pairs account for $90 \%$ and $3 \%$ of the total fluctuating kinetic energy, respectively. Thus, the flow dynamics is dominated by mode pair I. The evolution of their temporal coefficients is given in figures ??(a,b), which have the same content except for a phase shift of $\pi / 2$. This can also be validated by the Lissajous curves as shown in figures ??(a,b). The power spectral density of the harmonic mode coefficient shown in figures ??(a,b) demonstrate that their spectra have a peak at Strouhal numbers 0.23 and 0.46 , corresponding to frequencies of $24 \mathrm{~Hz}$ and $48 \mathrm{~Hz}$, respectively. In comparison, analysis of experimental data for this system demonstrated coherent structures with Strouhal number at 0.27 both by POD of the pressure fields [? ] and by phase averaging of the velocity fields [? ]. Further investigations of the flow fields by SPOD showed similar results, i.e., the SPOD modes have Strouhal numbers of 0.27 and 0.54 [? ].
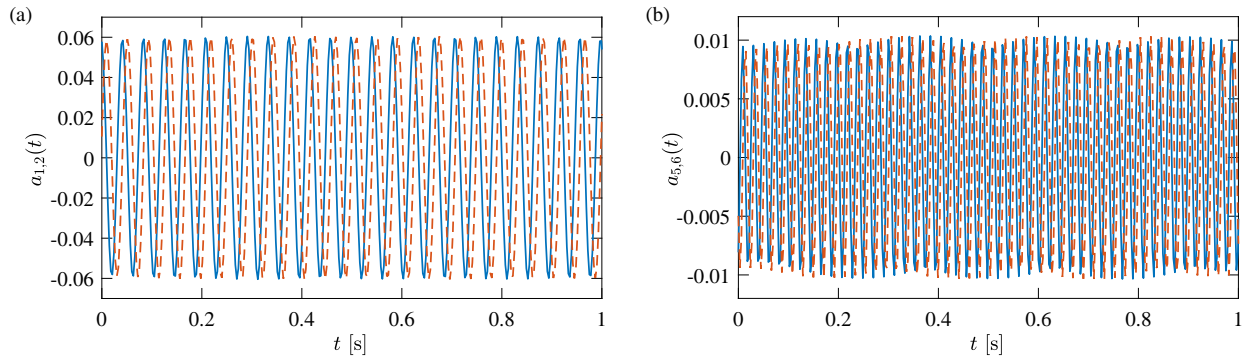

Figure 9: Evolution of the temporal coefficients of the first (a) and third (b) energy-ranked SPOD mode pairs.
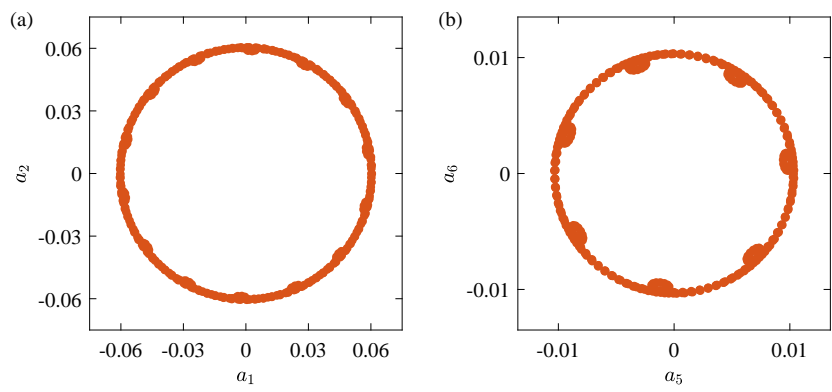

Figure 10: Phase portrait of the temporal coefficients of energy-ranked mode pair I (a) and III (b). 

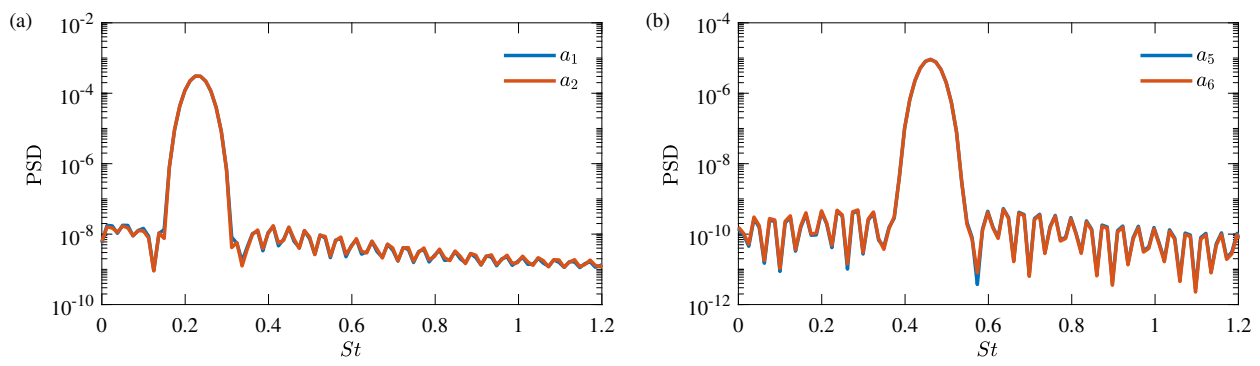

Figure 11: The power spectral density (PSD) of the first (a) and third (b) energy-ranked SPOD mode pairs.
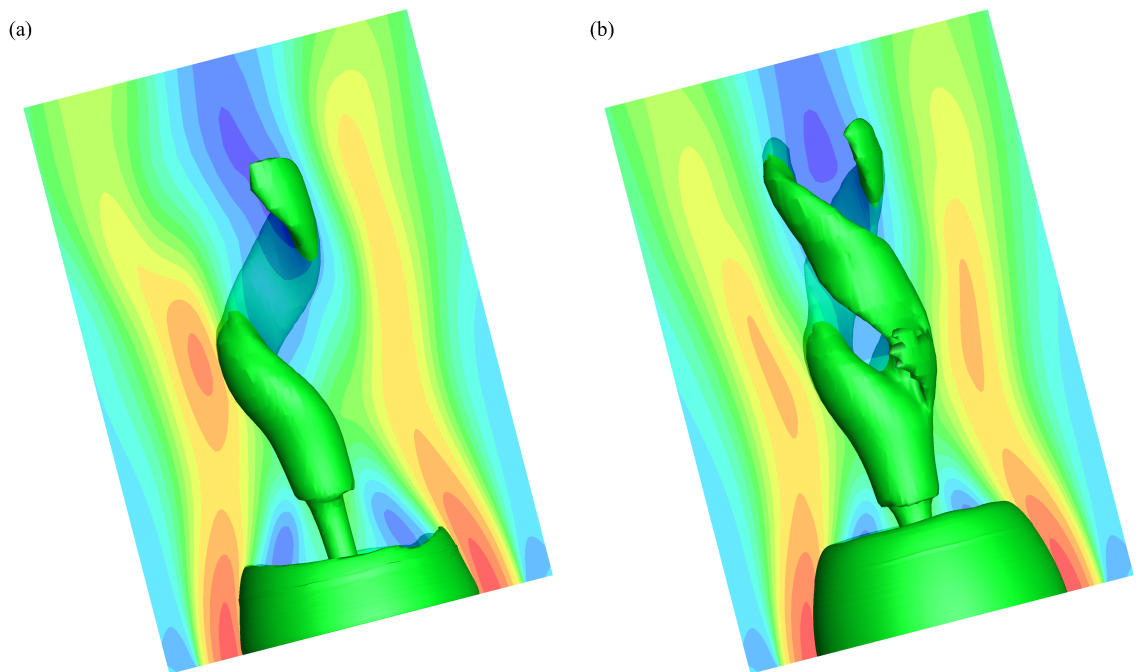

Figure 12: Reconstructed velocity field with the first (a) and third (b) energy-ranked mode pair: slices with contours of axial velocity in $x-y$ plane; and $Q$ criterion vortices visualization with isosurfaces of positive $Q=5 \times 10^{4} 1 / s^{2}$ (a) and $2.5 \times 10^{4} 1 / s^{2}$ (b), respectively.
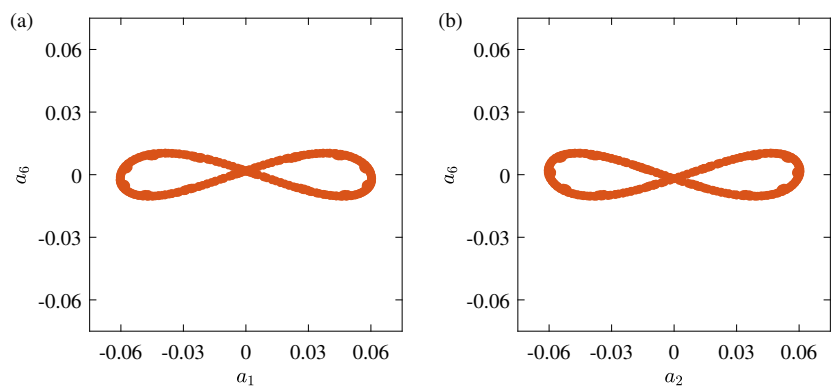

Figure 13: Phase portrait of the temporal coefficients between mode pair I (a) and III (b). 


\subsection{Single and double helical structures}

The reconstructed velocity fields are presented in figures ??(a,b). Here, the $Q$ criterion [? ]

$$
Q=\frac{1}{2}\left(\|W\|^{2}-\|S\|^{2}\right)
$$

is applied to identify vortical structures in the flow field. The vorticity tensor $W_{i j}$ and rate-of-strain tensor $S_{i j}$ are defined by

$$
W_{i j}=\frac{1}{2}\left(\frac{\partial u_{i}}{\partial x_{j}}-\frac{\partial u_{j}}{\partial x_{i}}\right)
$$

and

$$
S_{i j}=\frac{1}{2}\left(\frac{\partial u_{i}}{\partial x_{j}}+\frac{\partial u_{j}}{\partial x_{i}}\right)
$$

respectively. The results reveal that not only is there a single helical vortical structure, but also a double helical structure, and they both correspond to the spiral type of vortex breakdown. These spiral-shaped structures have high vorticity wrapped around the vortex breakdown bubble. It should be noted that the double helix structure is rarely observed in swirling jets, and the double structure is in line with that detected in turbulent annular swirling jet by the phase average method [? ]. Further analysis illustrates that the double helix structure is the second order harmonic mode of the single one just as indicated by the Lissajous curves in figures ??(a,b). This result is different from the experimental study by Vanierschotet al. [? ] that these structures are not higher harmonics of each other. They also performed global stability analysis of the mean flow fields and revealed that the single and double helix structures correspond to two separate global modes [? ]. The influence of the single and double helix on the second order statistics is shown in figure ??. Comparing with figure ?? shows that these fluctuations are in the same order of magnitude compared to the turbulent fluctuations, especially for the single helix. This illustrates the large influence of these coherent structures on the dynamics of the flow field. The distribution of the single helix shows large values of $\tilde{u}_{x} \tilde{u}_{x}$ and $\overline{\tilde{u}}_{z} \tilde{u}_{z}$ near the central axis, as also shown in the experimental results presented by Vanierschot et al. [? ]. The other fluctuations are situated on the boundary of the vortex breakdown bubble and the jet, which is also the case in the experimental measurements. 

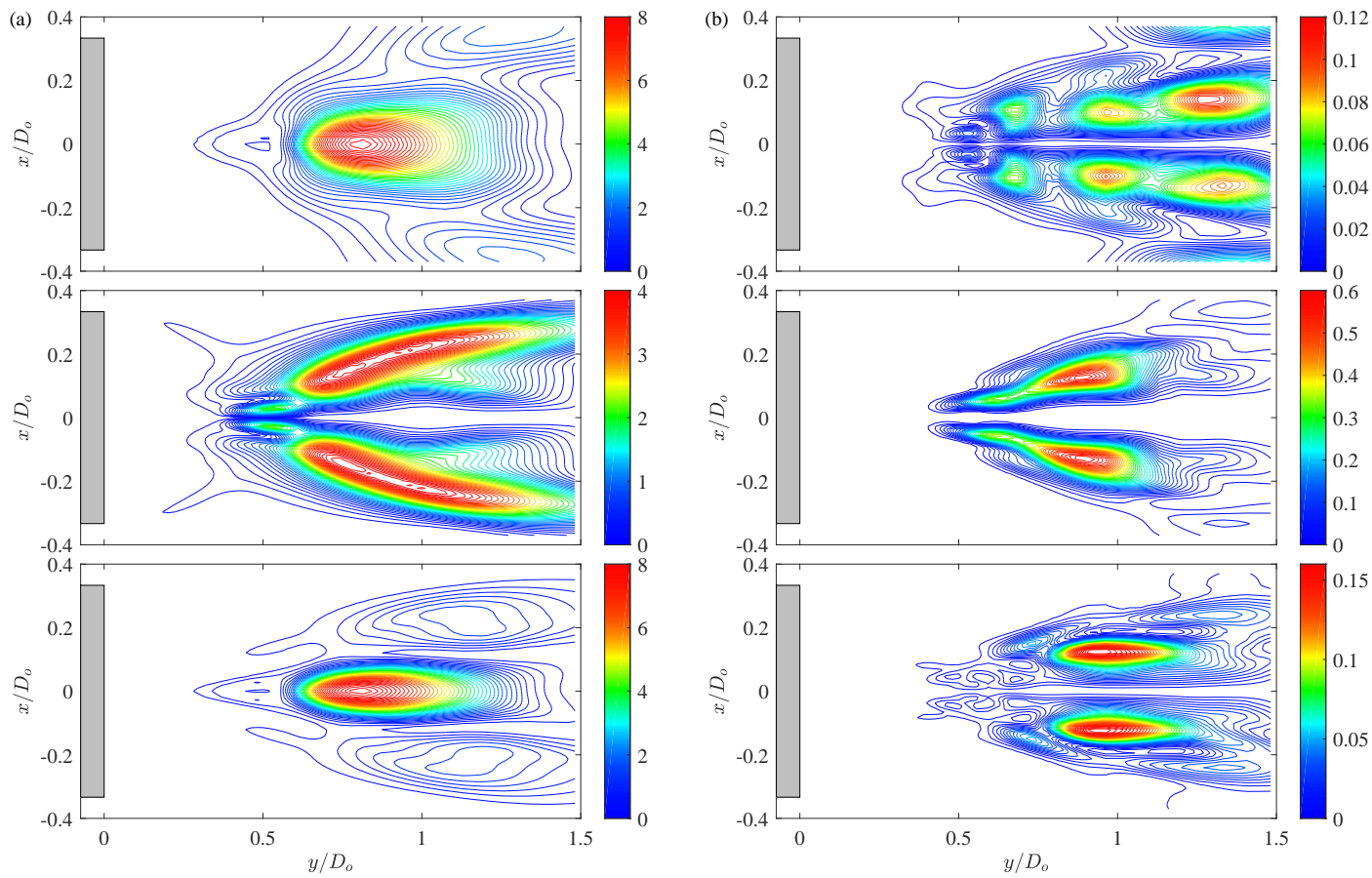

Figure 14: Contours of the normal coherent stresses introduced by the single (a) and double (b) helical structures: $100 \times$ $\left\langle\overline{\tilde{u}_{x} \tilde{u}_{x}}\right\rangle / U_{0}^{2}, 100 \times\left\langle\overline{\tilde{u}_{x} \tilde{u}_{x}}\right\rangle / U_{0}^{2}$ and $100 \times\left\langle\overline{\tilde{u}_{x} \tilde{u}_{x}}\right\rangle / U_{0}^{2}$ from top to bottom.

\section{Summary and conclusions}

In this paper, a turbulent annular swirling jet experiencing vortex breakdown is simulated for Reynolds number $R e=8500$ and swirl number $S=0.39$ by the unsteady Reynolds-averaged Navier-Stokes approach, where the SSG Reynolds stress turbulence model is used. The flow topology is representative for an annular swirl combustor. The time-averaged flow fields calculated from the numerical simulation are in reasonable agreement with experimental results. Moreover, regardless of the frequency shift arising from the inexact prediction of velocity profiles just upstream of vortex breakdown, the precession frequency of the precessing vortex core is predicted with reasonable accuracy (the difference is less than 14\%). Furthermore, the spectral proper orthogonal decomposition methodology is applied to investigate the coherent structures in the flow field. It is found that, apart from the single helical vortical structure usually reported in turbulent swirling flows, a double helical vortical structure is also identified. These single and double spiral type coherent structures with excessive vorticity are wrapped around the vortex breakdown bubble and rotating in the counter-swirl direction for Strouhal numbers 0.23 and 0.46 , respectively. This result has good agreement with the recent experimental observations by Vanierschotet al. [? ] (with a discrepancy of 11\%). The findings in this work indicate that the unsteady Reynolds stress model can predict the periodic large scale 
coherent structures in turbulent annular swirling jet flow both temporally and spatially with good accuracy.

\section{Acknowledgements}

This work was supported by the China Scholarship Council (CSC) under Grants No. 201706890016. The simulations were carried out on the Tier-2 cluster of the Flemish Supercomputer Center (Vlaams Supercomputer Centrum - VSC). 